\title{
Natural Energy Bars with Protein Improvement From Animal Origin Foods
}

\section{Barras Energéticas Naturales con Mejoramiento Proteico a Partir de Alimentos de Origen Animal}

\author{
M. A. Logroño Veloz, S. L. Betancourt Ortiz, and J. G. Fonseca
}

Carrera de Nutrición y Dietética, Facultad de Salud Pública, Escuela Superior Politécnica de Chimborazo, Riobamba, Ecuador

VII International Congress of

Science, Technology,

Entrepreneurship and

Innovation (SECTEI 2020)

Corresponding Author:

M. A. Logroño Veloz

mlogrono@espoch.edu.ec

Published: 26 August 2021

Production and Hosting by

Knowledge E

(c) M. A. Logroño Veloz

et al. This article is distributed under the terms of the Creative Commons

Attribution License, which permits unrestricted use and redistribution provided that the original author and source are credited.
S OPEN ACCESS

\section{Abstract}

The energy bars provide the consumer with nutritional and organoleptic quality, as well as a prolonged shelf life without the need to modify the temperature for storage, however, they do not satisfy the nutritional requirements of high-quality proteins since they are usually made from cereals, which are low cost ingredients and great energy contribution. The objective of this study was to create an energetic and nutritious bar, without diminishing the technological quality of the commercial bars and that, due to their sensory characteristics, are easily included in the daily consumption of people who exercise and want to control their weight. A product was formulated with a greater contribution and protein quality, and with a functional dose of macronutrients. The product was prepared in compliance with the regulations of the Ecuadorian Institute for Standardization (INEN) and the Food Codex, the premixing and roasting of ingredients was controlled and finally food with animal protein source such as egg white and powdered milk was added. For the premix oat flakes, nuts such as nuts, almonds and hazelnuts, chia seeds and honey were used, then added egg white and powdered milk. The protein content it reached was $29.01 \%$ on a wet basis, a fat value of $23.10 \%$ and carbohydrates of $25.24 \%$ on $100 \mathrm{~g}$ of sample on a wet basis. The energy distribution of macronutrients was balanced, and the sensory evaluation showed good product acceptability.

Keywords: cereal bars, protein, immediate energy.

\section{Resumen}

Las barras energéticas proporcionan al consumidor calidad nutricional y organoléptica, así como una vida de anaquel prolongada sin necesidad de modificar la temperatura para su almacenamiento, sin embargo, no satisfacen los requerimientos nutricionales de proteínas de alta calidad ya que generalmente están elaboradas a base de cereales, que son ingredientes de bajo costo y gran aporte energético. El objetivo se este estudio fue crear una barra energética y nutritiva, sin disminuir la calidad tecnológica de las barras comerciales y que por sus características sensoriales sean fácilmente incluidas en el consumo diario de personas que se ejercitan y quieren controlar su peso. Se formuló un producto con un mayor aporte y calidad proteica, y con una dosis funcional de macronutrientes. El producto se elaboró cumpliendo las normativas del Instituto Ecuatoriano de Normalización (INEN) y del Codex alimentario, se controló la premezcla y tostado de ingredientes y finalmente se adicionó los alimentos con fuente proteica animal como la clara de huevo y leche en polvo. Para la premezcla se utilizaron hojuelas de avena, frutos secos como nueces, almendras y avellanas, semillas de chía y miel de abeja, luego se adicionó la clara de huevo y la leche en polvo. El contenido proteico que

How to cite this article: Veloz MAL, Ortiz SLB, Fonseca JG (2021). Natural Energy Bars with Protein Improvement From Animal Origin Foods. ESPOCH 
alcanzó fue de $29,01 \%$ en base húmeda, un valor en grasas de $23,10 \%$ y de carbohidratos de $25,24 \%$ de muestra en base húmeda. La distribución energética de macronutrientes fue equilibrada y la evaluación sensorial arrojó una buena aceptabilidad del producto.

Palabras Clave: barras de cereales, proteína, energía inmediata.

\section{Introducción}

Según el informe de costumbres y prácticas deportivas en la población ecuatoriana [1], el $41,8 \%$ de la población de 12 años y más, practica deporte regularmente. Existe una concienciación y prevención en la mente de los ecuatorianos de verse y sentirse bien, es por esto por lo que muchas más personas se suman a esta tendencia de estar en forma y practican deportes como: crossfit, pilates, yoga, zumba, running, artes marciales, etc. [2]. Para una persona que practica deportes o rutinas de ejercicios regularmente es muy importante consumir una dieta balanceada que le aporte una cantidad de energía y nutrientes necesarios para mantener y reparar los tejidos musculares, y un buen nivel de metabolismo corporal. El nutriente indispensable para lograr los objetivos de este grupo de población es la proteína, macronutriente que permite una recuperación muscular más rápida, contribuyendo al crecimiento y aumento de calidad muscular y por lo tanto a un mejor rendimiento [3].

En el mundo actual, al analizar la alimentación de la población que se ejercita y quiere controlar su peso, ya no basta considerar únicamente los alimentos, sino que se debe tener presente otras alternativas cuando los requerimientos de nutrientes son elevados, tales como los suplementos nutricionales (SN), su ingesta es común entre las personas que se ejercitan con la finalidad de mantener y mejorar la capacidad y el rendimiento físico [4], sin embargo éstos muchas veces son utilizados como complemento a la dieta, sin mayor respaldo científico que justifique su uso.

Las barras energéticas son productos que cada vez van tomando auge en la población que se ejercita para obtener energía inmediata, sin embargo la composición de macronutrientes de las barras de cereales comerciales no presentan características particulares que justifiquen su posicionamiento como productos saludables o de buena calidad nutricional ya que contienen baja cantidad de proteínas y de pobre calidad por provenir casi exclusivamente de cereales (arroz, avena, maíz), siendo el aporte proteico por unidad de barra escaso (tan sólo 5,5\%) [5], es por este motivo que se propone crear una barra energética que se compare con los mismos parámetros que tienen los suplementos creando un producto con alto contenido de proteína, fibra, excluyendo ácidos grasos trans para evitar algún efecto secundario que afecte el 
rendimiento o cause algún tipo de daño en el organismo y que se ingieran como un complemento alimentario por su alto aporte nutricional como carbohidratos, proteína y grasa saludables, además que se categorice como funcional por incluir una gran variedad de granos enteros, cumpliendo con la tendencia actual de utilizar productos propios de cada zona y mucho más saludables [6].

Un alimento con un alto aporte proteico es la clara de huevo con un $12 \%$ aproximadamente de contenido de proteína, tiene funciones importantes como ayudar a equilibrar el potasio y el sodio, necesarios para mantener el potencial de la membrana, interviene en la contracción muscular, la transmisión del impulso nervioso, la función cardíaca, transferencia de nutrientes y metabolitos a través de la célula [7], contiene los 9 aminoácidos esenciales, principalmente la ovoalbúmina, la lisozima, ovotransferrina y la ovomucina, es fuente de vitamina B2 y niacina [8], sus factores anti nutricionales (inhibidores de la tripsina y la avidina que puede ligar la biotina), son termolábiles [9].

También se puede destacar la leche en polvo como otro alimento con un alto aporte de macro y micronutrientes esenciales, ya que a pesar de presentarse deshidratada contiene todos los componentes naturales de la leche normal con un alto valor energético, elevado valor proteico y una proporción muy alta de calcio, fundamental para el tejido óseo y sin conservantes [10, 11].

Se debe tomar muy en cuenta que la proteína no es conveniente utilizarla como una fuente de energía debido a que aumentaría los niveles de requerimientos y al no cubrirlos se daría una pérdida de proteína endógena, lo cual reduciría el rendimiento deportivo y causaría sobre entrenamiento, llegando a reducir la masa y la fuerza muscular, por lo que es recomendable que se mantenga un balance entre proteína con carbohidratos, principalmente [12, 13].

El objetivo del presente trabajo fue elaborar una barra energética natural con mejoramiento proteico a partir de alimentos de origen animal como la clara de huevo y la leche en polvo, logrando una funcionalidad entre macronutrientes y que además tenga una evaluación sensorial óptima que motive integrarla en el consumo diario de las personas que se ejercitan.

\section{Materiales y Métodos}

Se trató de un estudio experimental con eje descriptivo de tipo cuali- cuantitativo, para el diseño experimental se consideró un tratamiento blanco (T1) y un tratamiento control (T2); en el tratamiento blanco no se usaron productos de origen animal como la clara de huevo y la leche entera en polvo. La elaboración de los productos tuvo una duración de 3 meses, se realizó en el laboratorio de Tecnología de los alimentos de la Carrera de Nutrición y Dietética de la Escuela Superior Politécnica de Chimborazo (ESPOCH), se trabajó cumpliendo todas las normas de Buenas Prácticas de Manufactura (BPM) 
del Ministerio de Salud Pública [2], INEN y Codex alimentario para barras energéticas, para obtener un producto de buenas características organolépticas y principalmente de calidad [14-16].

\subsection{Preparación de la barra energética}

El diagrama de flujo que resume las etapas del proceso de elaboración se presenta en la Figura 1. Se realizaron muestras previas para encontrar la composición y características organolépticas adecuadas del producto. Para la muestra final se utilizaron: Hojuelas de avena, frutos secos: Avellanas, nueces, almendras, semillas: Chía, frutas secas: Pasas y ciruelas pasas; edulcorante: Miel de abeja y se integraron la clara de huevo y la leche en polvo y como aromatizante: Canela. La mezcla de todos estos productos se sometió a tostado a fuego lento por un tiempo de $10 \mathrm{~min}$. Se distribuyó en el papel de cocina uniformemente para darle la forma de barra con un ancho de $2,5 \mathrm{~cm}$ y un largo de $10 \mathrm{~cm}$. Las condiciones de enfriamiento fueron a $4^{\circ} \mathrm{C}$ durante 15 min para finalmente hornear por $15 \mathrm{~min}$ a una temperatura de $280^{\circ} \mathrm{C}$.

Se realizó un análisis sensorial por medio de una prueba de aceptabilidad a 45 personas que acudían con regularidad al gimnasio, se usó para cada degustador, muestras de la barra energética en porciones de aproximadamente $10 \mathrm{~g}$, la escala hedónica tuvo 5 categorías y se asignó a cada categoría, un número del uno al cinco: 1 Me disgusta extremadamente, 2 Me disgusta moderadamente, 3 No me gusta ni me disgusta, 4 Me gusta moderadamente, 5 Me gusta extremadamente. Los atributos para investigar fueron apariencia, color, textura, aroma y sabor. Los números asignados se tabularon y analizaron determinando el promedio y la desviación estándar.

Los análisis bromatológicos se ejecutaron en el laboratorio de Bromatología de la ESPOCH, se usó el programa InfoStat/L 2018, para determinar significancia en relación con los tratamientos, también se realizaron análisis en el Laboratorio de Control y Análisis de Alimentos (CONAL).

Las determinaciones de humedad, cenizas, acidez titulable, $\mathrm{pH}$, fibra, carbohidratos, azúcares reductores se realizaron según la metodología que se indica a continuación:

La humedad se determinó mediante el desecado en estufa por circulación de aire caliente de acuerdo con las normas NTE INEN-ISO 712 [17].

La determinación de ceniza fue mediante la incineración en mufla a una temperatura de $550^{\circ} \mathrm{C}$ basado en las normas NTE INEN 520. El contenido fibra se determinó a través de la extracción mediante digestor guiado por las normas NTE INEN 0523.

El contenido de carbohidratos se realizó mediante la reacción y oxidación de Fehling, a partir de la hidrolización con ácido clorhídrico y con la mezcla de 2 soluciones: Solución A (tartrato de Na y K en medio básico y Solución B (Sulfato cuproso). 
Los azúcares reductores se obtuvieron por medio de titulación por oxidación de Fehling a partir de dos soluciones: Tartrato de Na y K en medio básico (Solución A) y Sulfato cuproso (Solución B).

El análisis de grasa se realizó por el método de extracción continua con Éter por el método de Goldfish.

Para la determinación de proteína se elaboró el análisis por el método de Kjeldahl. Todos los análisis se realizaron por triplicado y los resultados se expresaron en g por $100 \mathrm{~g}$ de muestra.

\section{Resultados y Discusión}

\subsection{Aceptabilidad de las barras energéticas}

Los productos obtenidos mostraron buen aspecto, color dorado, textura mixta, crocante y con un aroma muy agradable a canela; presentaron mayor aceptabilidad los atributos del T2; la textura, se debe a la adición de leche y clara de huevo que ayuda a dar firmeza y a que se compacten mejor los ingredientes y el color mejora por las posibles reacciones de Mayllard entre la lactosa y las proteínas del huevo que no contiene el T1.

\subsection{Análisis físicos de los tratamientos de la barra energética}

A nivel de industria alimentaria uno de los principales factores que afectan al crecimiento bacteriano es el pH medido en términos de acidez, el mismo que es trascendente para regular el riesgo microbiológico; la adición de leche en polvo en el T2 aumenta el $\mathrm{pH}$, cabe recalcar que valores de $\mathrm{pH}$ entre 6 y 8 aumenta el riesgo de contaminación bacteriana, las barras elaboradas están bajo el rango que no implica peligro, pero debe cuidarse la contaminación por mohos, por lo tanto los productos deben ser almacenados en ambientes secos para garantizar seguridad alimentaria.

\subsection{Análisis bromatológico de la barra energética}

La composición bromatológica básica de las barras es sustancial para establecer las cantidades de macronutrientes como proteínas, carbohidratos, grasa, fibra y para diferenciar el aporte significativo de la adición de semillas de chía, leche y clara de huevos entre el tratamiento de control y el blanco; además, es información esencial para elaborar las recomendaciones de valor diario en una etiqueta nutricional. 


\subsubsection{Humedad}

La humedad de los tratamientos no presentó diferencias significativas, debido a que fueron sometidos los ingredientes a un proceso de tostado, para compactarlos. Se obtuvo un valor promedio menor a $10 \%$, valor reconocido como adecuado para la conservación de este tipo de productos, ya que no permite el crecimiento microbiano ni fúngico [18].

\subsubsection{Ceniza}

Existe diferencias significativas debido a que el T2 contiene clara de huevo además de los frutos secos y semillas. El T2 tiene un valor de ceniza de 1,03\%, la literatura evidencia que hay barras de cereales con contenidos que van desde 5,09\% hasta $1,18 \%$ de cenizas, aquellas barras de cereales que además tenían un alto valor proteico arrojaron un porcentaje de cenizas de 2,2 , la diferencia de ingredientes y formulaciones determinan la divergencia de estos valores [19].

\subsubsection{Fibra}

Existe una diferencia significativa entre en T1 y T2, ya que en el T2 se añadió semillas de chía que contiene fibra dietética, la cual ayuda a regularizar el tránsito intestinal, reduce los lípidos, la glucemia en diabéticos, entre otros beneficios [20]. Otro ingrediente rico en fibra fue la avena, estas hojuelas contienen betaglucano, numerosos estudios señalan que el consumo de un mínimo de $3 \mathrm{~g} / d$ ía de betaglucano de forma regular, como parte de una dieta con un bajo contenido en grasa saturada y colesterol, puede contribuir a disminuir el riesgo de enfermedad coronaria, tal y como han aprobado diversas agencias reguladoras, como la Food and Drug Administration de Estados Unidos o la Agencia Europea de Seguridad Alimentaria [21].

\subsubsection{Azúcares reductores}

No existe significancia entre los dos tratamientos, ya que se ha utilizado la misma cantidad de endulzante que es la miel de abeja. Los azúcares en la miel son los componentes más importantes, éstos determinan sus atributos físicos, según su clase y concentración, la glucosa y fructosa son sus azúcares reductores en un 90\% [22]. Es importante resaltar las virtudes de este edulcorante como bactericida natural en heridas infectadas, así como su actividad antioxidante, antiinflamatoria y de desbridamiento [23], otorgando una característica nutraceútica a los prototipos propuestos. 


\subsubsection{Carbohidratos totales}

Al comparar los dos tratamientos de la barra energética, se establece que no existió diferencias estadísticamente significativas entre el T1 y T2. Los ingredientes incluidos en los dos tratamientos, a excepción de la clara de huevo, contienen carbohidratos simples y complejos [24].

\subsubsection{Grasa}

Los tratamientos presentaron un alto aporte de grasas, no hubo diferencias significativas entre el T1 y $\mathrm{T} 2$. Los productos industrializados suelen contener grasas trans, colorantes, saborizantes y azúcares, ofertando al consumidor productos de buen sabor pero de bajo valor nutritivo, que son consumidos masivamente por la población, lo que ocasiona un aporte elevado de energía en la dieta [1]. T1 y T2 tienen entre sus ingredientes avena, que es muy rica en contenido de lípidos, contiene más del $10 \%$ de lípidos en comparación con aproximadamente el 2-3\% en el trigo y en la mayoría de los demás cereales (en el maíz, alrededor del 17\%), la lecitina es su principal fosfolípido (45-51\%) [25]. Otra fuente de grasa fue la Chía, que contiene ácidos grasos de cadena hidrocarbonada larga, destacando el palmítico (C16), esteárico (C18), oleico (C18:1), linoleico (C18:2) y alfa- linoleico (alfa-C18:3) [26], que combinada con los ácidos grasos de los frutos secos (omega 6), contribuirían al equilibrio entre omega 3 y omega 6 necesario para la prevención y tratamiento de las enfermedades cardiovasculares [27] (1\% de omega 3, así como 4\% de omega 6) [28].

\subsubsection{Proteína}

Existieron diferencias significativas entre T1 y T2 ya que en T2 se adicionó leche en polvo y clara de huevo, aumentando así el aporte proteico. Se ha demostrado que la utilización de proteínas lácteas, como ingredientes para la formulación de barritas energéticas tiene un marcado efecto hipoglucemiante, con valores de índice y carga glucémica significativamente menores que los que presentan las barritas elaboradas con hidratos de carbono. Asimismo, estas barritas elaboradas con proteínas lácteas promueven una sensación del efecto de saciedad mucho mayor y durante más tiempo que las elaboradas con carbohidratos, lo que pone de manifiesto el potencial de estos ingredientes para la elaboración de alimentos con un alto valor dietético y nutricional [29]. 


\subsection{Análisis nutricional}

Se elaboró la información nutricional con una porción de 60 g, tomando como referencia una dieta de $2000 \mathrm{kcal}$. Se establecieron los siguientes valores para el tratamiento 2 que presento mayor aceptabilidad y mejor calidad bromatológica.

La elaboración de la barra energética tuvo como fin que el producto tenga un mayor aporte proteico de fuente natural y que las personas que se ejercitan y desean controlar su peso se beneficien de este aporte nutricional. En la formulación del producto se empleó un solo cereal básico con alto aporte nutricional como la avena, pero complementado con diferentes tipos de frutos secos que son fuentes de grasa y de proteína, además la adición de clara de huevo y leche en polvo que permitieron mejorar su aporte nutricional, el producto usa la miel de abeja como endulzante, a diferencia de las Barras Comerciales (BC) que son elaboradas principalmente con cereales como avena arroz, maíz y con un alto contenido de azúcares a partir de jarabe de maíz, glucosa o azúcar [29].

Se logró una mejora del aporte proteico gracias a la inclusión de fuentes de proteína animal: Leche en polvo y clara de huevo, que son una excelente fuente de proteínas de alta calidad, alcanzando un 29,01\%, las BC con aporte de clara de huevo reportan un contenido proteico de $21,6 \%$ [30], y otras llegan a $17,40 \%$ de aporte en proteína. Arruti et al. valores por debajo del porcentaje obtenido [5].

El porcentaje de grasa fue de $23,10 \%$ éste está constituido principalmente por la grasa de los frutos secos y de la leche en polvo, los primeros tienen un alto contenido en ácidos grasos mono y poliinsaturados, como el ácido oleico, el linoleico (omega 6) y linolénico (omega 3) mientras que la grasa láctea contiene ácido linoleico conjugado (CLA), la esfingomielina, el ácido butírico y el ácido mirístico, que quizá podrían brindar protección contra enfermedades crónicas graves, además la grasa láctea podría tener un efecto beneficioso en la salud de los huesos, según algunos estudios experimentales realizados en animales [31, 32]. Otras BC contienen hasta un $12,6 \%$ de grasa proveniente de mantecas y aceites vegetales hidrogenados, aportando ácidos grasos trans con efectos nocivos para el consumidor que van desde el aumento del riesgo de padecer cáncer hasta el padecimiento de dislipidemia [33].

En cuanto a su aporte de grasa por poción, otros productos presentan 2,8 $\mathrm{g}$ en tanto que la barra energética elaborada quintuplica este valor (13,86 g) [33], el producto ofrece un alto valor nutricional basado únicamente en fuentes naturales.

Las BC están categorizadas a nivel comercial como alimentos saludables por la inclusión de granos enteros principalmente, lo que determinaría un incremento en el aporte de fibra, sin embargo, los porcentajes de fibra dietaría de las BC son bajos, entre 1,0 y $6 \%$, por lo cual no presentan grandes aportes al consumidor, mientras que la barra elaborada contiene $22,58 \mathrm{~g}$ de fibra $[34,35]$. 


\section{Conclusiones}

La formulación de las Barras Energéticas contiene ingredientes de origen animal: Leche en polvo y clara de huevo, productos naturales, que permitieron obtener un producto con un mayor aporte proteico y sin el uso de ningún aditivo.

El producto cuenta, con un buen aporte de macronutrientes, en los porcentajes recomendados para lograr que la persona que la consuma obtenga energía y resistencia en los entrenamientos, los ingredientes incluidos para la elaboración de la Barra Energética aportan además minerales que no pueden ser sintetizados por el organismo lo que ayudará a reponer al cuerpo de electrolitos perdidos en el sudor durante el entrenamiento. La Barra Energética tuvo buena aceptabilidad por sus características organolépticas.

\section{Conflicto de Intereses}

Las autoras declaran que no existe conflicto de intereses que puedan afectar directa o indirectamente los resultados obtenidos.

\section{References}

[1] INEC. Costumbres y prácticas deportivas en la población ecuatoriana. Quito; 2009.

[2] ARCSA 067. Normativa técnica sanitaria para alimentos procesados, plantas procesadoras de alimentos, establecimientos de distribución, comercialización, transporte y establecimientos de alimentación colectiva. Quito; 2015.

[3] Morán P. Factores que inciden en el consumo de barras energéticas para deportistas en la ciudad de Guayaquil. [actualizado marzo 2016; citado 5 enero 2020]. Disponible en: http://192.188.52.94:8080/bitstream/3317/6157/1/ T-UCSG-POS-MGM-33.pdf

[4] Grupo de Trabajo sobre Nutrición del Comité Olímpico Internacional. Nutrición para deprotistas. 2012. Recuperado el 16 de abril de 2019, de Nutrición para deprotistas: http://deporte.aragon.es/recursos/files/documentos/doc-areas_ sociales/deporte_y_salud/guia_nutricion_deportistas.pdf

[5] Arruti I, Fernández M, Martínez R. Diseño y Desarrolllo de uan Barra Energética para Deportistas de Triatlón. Universidad Católica de Uruguay; 2015.

[6] Martínez J, Urdampilleta A, Mielgo J. Necesidades energéticas, hídricas y nutricionales en el deporte. European Journal of Human Movement. 2013;10(17).

[7] Baltensperge D. Release of Plainsman. 1991; 4(4):7. 
[8] Berti MRS, Wickens R, Figueroa I. Field evaluation of grain amatanth in Chile. Chile: Janick; 1997.

[9] Bocci D, Casas M. Producción de Leche en Polvo Entera, Parcialmente Descremada y Descremada. 2013 September Available from: http://bdigital.uncu.edu.ar/objetos_ digitales/7878/producciondelecheenpolvo.pdf

[10] Bonet B, Dalmau J, Gil I et al. Productos Lacteos Insustituibles. Recuperado el 28 de abril de 2019, de Leche, Nata, Mantequilla y otros productos lácteos: http://www.lacteosinsustituibles.es/p/archivos/pdf/monografia_leche_ nata_mantequilla_otros.pdf

[11] Camera K. Camera, Come bien, Corre mejor: Guía para entenar tu alimentaciñon y optimizar el rendimiento. Argentina: Penguin Random House Group; 2016.

[12] Carrión M, Giacomino S, Pellegrino N, Sambucetti M. Composición y Perfil Nutricional De Barras De Cereales Comerciales. La Academia. 2009;10.

[13] Dominguez R. Necesidades proteicas en el deportista de resistencia. 2013.

[14] FAO. Caracterización y efecto de la densidad en el cultivo de amaranto. Rome: FAO; 1991. Available from: http://www.fao.org/tempref/Gl/Reserved/FTP_FaoRIc/old/prior/ segalim/prodalim/prodveg/cdrom/contenido/libro01/Cap10.htm

[15] Grupo de Trabajo de Nutrición del Comité Olímpico Internacional. Nutrición para deportistas. 2012. Available from: http://deporte.aragon.es/recursos/files/ documentos/doc-areas_sociales/deporte_y_salud/guia_nutricion_deportistas.pdf

[16] Haymes E. (2008). Sumpplementation to athletes. Sports Nutr, 146-69.

[17] Norma Técnica Ecuatoriana NTE INEN 20852005 (Ecuador).

[18] León ME. Nutricional Y Sensorial De Barras De Cereal Con Quinoa. Scielo. 2017;34(li):33-43.

[19] Marchese NR, Novello Z. Desenvolvimento e caracterização de barra de cereal salgada. Rev Bras Tecnol Agroindustrial. 2017;11(1):2150-64.

[20] Carrillo-Gómez CS, Gutiérrez-Cuevas M, Muro-Valverde M, Martínez- Horner R, Torres-Bugarín O. La chía como súper alimento y sus beneficios en la salud de la piel. El Resid. 2017;12(1):18-24.

[21] Vizuete AA, Anta RMO. Effects of oat beta-glucan intake on blood cholesterol: A review. Rev Esp Nutr Humana y Diet. 2016;20(2):127-39.

[22] Urrego RJF. Carecterizacion de Mieles de Apis mellifera, Colectadas De Diferentes Regiones de Antioquia. Univ Nac Colomb. 2017;83.

[23] Schencke C, Vásquez B, Sandoval C, Del Sol M. El Rol de la Miel en los Procesos Morfofisiológicos de Reparación de Heridas. Ann Oncol. 2020;( January).

[24] Aponte Guevara C, Sánchez Guzmán G, Muguruza Crispin N, Chiroque Sernaqué D, Guerrero Romero R, Carreño Mundo H. Elaboración y aceptabilidad de barritas energético-proteico. Big Bang Faustiniano. 2017;6:36-41. 
[25] Montoya YH. Formulación de una barra energética con alta capacidad antioxidante dirigida a ciclistas recreativos. 2018;10. Available from: http://dx.doi.org/10.1053/j.gastro.2014.05.023\{\%\}0Ahttps://doi.org/10.1016/j.gie. 2018.04.013\{\%\}OAhttp://www.ncbi.nlm.nih.gov/pubmed/29451164\{\%\}0Ahttp: //www.pubmedcentral.nih.gov/articlerender.fcgi?artid=PMC5838726\{\%\}250Ahttp: //dx.doi.org/10.1016/j.gie.2013.07.022

[26] Tolentino RG, Vega MLR, Vega y León S, Fontecha J, Rodríguez LM, Medina AE. Fatty acid content in chia (Salvia hispanica L.) seeds grown in four Mexican states. Rev Cuba Plantas Med. 2014;19(3):199-207.

[27] Gómez Candela C, Bermejo López LM, Loria Kohen V. Importancia del equilibrio del índice omega-6/omega-3 en el mantenimiento de un buen estado de salud. recomendaciones nutricionales. Nutr Hosp. 2011;26(2):323-9.

[28] Reyna N, Moreno Rojas R, Mendoza L et al. Formulación de barras nutricionales con proteínas lácteas: Índice glucémico y efecto de saciedad. Nutr Hosp. 2016;33(2):395-400.

[29] Instituto de Estudios del Huevo. El huevo en la Dieta y la Salud. 2018. Recuperado el 28 de abril de 2019, de El huevo en la Dieta y la Salud: https://www.institutohuevo. com/el-huevo-en-la-dieta-y-la-salud/

[30] Moore DCD. Mas Allá de la Hipertrofia Muscular: Por Qué Son Importantes las Proteínas de la Dieta Para los Atletas de Resistencia. 2015. Recuperado el 18 de marzo de 2019,: https://g-se.com/mas-alla-de-la-hipertrofia-muscular-por-que-son-i mportantes-las-proteinas-de-la-dieta-para-los-atletas-de-resistencia-1894-sa-357c fb2725f563

[31] Olivera M, Giacomino S, Pellegrio N, Sambucetti M. Composición y perfil Nutricional de Barras de Cereales Comerciales. 2009;1(11).

[32] Olivera M, Ferreyra V, Giacomino S et al. Desarrollo de barras de cereales nutritivas y efecto del procesado en la calidad proteica. Revista Chilena de Nutricón. 2012;6(9).

[33] Palacios N, Montalvo Z, Ribas A. Alimentación, nutrición e hidratación en el deporte. 2009. Recuperado el 20 de marzo de 2019, de ALIMENTACIÓN, NUTRICIÓN E HIDRATACIÓN EN EL DEPORTE: https://www.cocacolaespana.es/content/dam/ journey/es/es/private/file-assets/nutricion/recomendamos/alimentacion-nutricion. pdf

[34] Sntana S. El huevo como aliado de la nutrición y salud. 2008. Recuperado el 28 de abril de 2019, de El huevo como aliado de la nutrición y salud: http://www. revicubalimentanut.sld.cu/Vol_18_2/Resumenes\{\%\}20Seminario\{\%\}2OHuevo.pdf

[35] Statista. Statistics anda facts on health. Fitness clubs. 2016. 